\title{
Synergistic anti-tumor effects of combined gemcitabine and cisplatin nanoparticles in a stroma-rich bladder carcinoma model
}

\author{
Jing Zhang ${ }^{\mathrm{a}, \mathrm{c}, 1}$, Lei Miao ${ }^{\mathrm{a}, 1}$, Shutao Guo ${ }^{\mathrm{a}}$, Yuan Zhang ${ }^{\mathrm{a}}$, Lu Zhang ${ }^{\mathrm{a}}$, Andrew Satterlee ${ }^{\mathrm{a}}$, \\ William Y. Kim ${ }^{b}$, and Leaf Huanga, ${ }^{a}$ \\ aDivision of Molecular Pharmaceutics and Center of Nanotechnology in Drug Delivery, Eshelman \\ School of Pharmacy, University of North Carolina at Chapel Hill, Chapel Hill, NC 27599, USA \\ bLineberger Comprehensive Cancer Center, University of North Carolina at Chapel Hill, Chapel \\ Hill, NC 27599, USA
}

'Key Laboratory of Modern Preparation of TCM, Ministry of Education, Jiangxi University of Traditional Chinese Medicine, Nanchang, Jiangxi 330004, China

\begin{abstract}
Tumors grown in a stroma-rich mouse model resembling clinically advanced bladder carcinoma with UMUC3 and NIH 3T3 cells have high levels of fibroblasts and an accelerated tumor growth rate. We used this model to investigate the synergistic effect of combined gemcitabine monophosphate (GMP) nanoparticles and Cisplatin nanoparticles (Combo NP) on tumorassociated fibroblasts (TAFs). A single injection of Combo NP had synergistic anti-tumor effects while the same molar ratio of combined GMP and Cisplatin delivered as free drug (Combo Free) fell outside of the synergistic range. Combo NP nearly halted tumor growth with little evidence of general toxicity while Combo Free had only a modest inhibitory effect at $16 \mathrm{mg} / \mathrm{kg}$ GMP and 1.6 $\mathrm{mg} / \mathrm{kg}$ Cisplatin. Combo NP increased levels of apoptosis within the tumor by approximately 1.3 fold (TUNEL analysis) and decreased a-SMA-positive fibroblast recruitment by more than $87 \%$ (immunofluorescence) after multiple injections compared with Combo Free, GMP NP or Cisplatin NP alone. The TAF-targeting capability of Combo NP was evaluated by double staining for TUNEL and a-SMA at various time points after a single injection. On day one after injection, $57 \%$ of the TUNEL-positive cells were identified as a-SMA-positive fibroblasts. By day four, tumor stroma was $85 \%$ depleted and $87 \%$ of the remaining TAFs were TUNEL-positive. Combo NP-treated tumors became 2.75 fold more permeable than those treated with Combo Free as measured by Evans Blue. We conclude that the antineoplastic effect of Combo NP works by first
\end{abstract}

\footnotetext{
(C) 2014 Elsevier B.V. All rights reserved.

*Corresponding Author: Division of Molecular Pharmaceutics and Center of Nanotechnology in Drug Delivery, Eshelman School of Pharmacy, University of North Carolina at Chapel Hill, Chapel Hill, NC 27599, USA. Tel.: +1 9198430736 ; fax: +1 9199660197. leafh@unc.edu.

1 These authors contributed equally to the work

Publisher's Disclaimer: This is a PDF file of an unedited manuscript that has been accepted for publication. As a service to our customers we are providing this early version of the manuscript. The manuscript will undergo copyediting, typesetting, and review of the resulting proof before it is published in its final citable form. Please note that during the production process errors may be discovered which could affect the content, and all legal disclaimers that apply to the journal pertain.
} 
targeting TAFs and is more effective as an anti-tumor therapy than Combo Free, GMP NP or Cisplatin NP alone.

\section{Keywords}

bladder cancer; stroma; gemcitabine; Cisplatin; tumor-associated fibroblasts

\section{Introduction}

Bladder cancer, the fourth most common cancer diagnosed in men and the eighth in women, remains a concern due to its prevalence and tendency to progress and recur [1-3]. Advances in drug delivery are needed and nanomedicine in particular holds promise as a means to improve bioavailability and half-life in circulation $[4,5]$. These therapies make use of the enhanced permeability and retention (EPR) effect in tumors, thereby increasing the chemotherapeutic dose to tumor tissue, while at the same time sparing normal tissue from exposure [6]. Improved drug uptake by tumor cells will also be an important consideration to achieve higher bioavailability.

Cancer has long been believed to be cell-autonomous and to date most research has focused directly on the malignant cells themselves. New data, however, suggests that carcinogenesis is determined not only by malignant cells, but also by a favorable tumor microenvironment [7]. Fibroblasts are the principal cellular component of the tumor microenvironment. Fibroblasts that have been recruited, activated and accumulated in close proximity to the tumor are referred to as tumor-associated fibroblasts (TAFs), myofibroblasts, or cancerassociated fibroblasts. In some instances, TAFs can be the most abundant type of cell within some tumors, notably pancreatic and breast carcinoma [8,9]. These cells are characterized by high expression of a-smooth muscle actin (a-SMA). They contribute to tumor growth and invasiveness by remodeling the extracellular matrix, secreting various soluble factors (growth factors and cytokines), and suppressing immune responses. Normalization or elimination of activated stroma should be considered as a potential approach to anti-cancer therapy [10]. Therapies directed at TAFs can be divided into four categories: (1) inhibition of stromal cell proliferation; (2) interference with growth factor/cytokine-mediated signals between TAFs and cancer cells [11]; (3) targeting of epigenetic alterations (DNA methylation); (4) modification of the inflammatory response [12].

Myofibroblasts tend to be present in invasive bladder tumors and may be an individual prognostic factor in urothelial carcinoma [13]. To identify and test TAF-targeting drugs for treatment of bladder cancer, a model system is needed which reproduces the stroma-rich tumor growth observed in patients. Human tumor xenografts in immunodeficient mice are often used for preclinical studies of anti-cancer agents. A subcutaneous xenograft is most commonly used due to ease of implanting tumor cells and unobstructed access to the tumor. A major criticism of this model, however, is that the local environment may not adequately simulate the carcinoma microenvironment [14]. Here we report the establishment of a stroma-rich, subcutaneous xenograft tumor model. Fibroblasts were subcutaneously coinjected along with cancerous cells and Matrigel, a permissive extracellular matrix 
preparation. When grown on Matrigel in vivo, bladder carcinoma cells adopt a more characteristic phenotype [15] and the xenograft better resembles the original tumor when compared to the more commonly used model.

We recently developed nanoparticles for treatment of non-small-cell lung cancer that encapsulate gemcitabine monophosphate (GMP, the bioactive form of gemcitabine) [16]. We have also developed Cisplatin nanoparticles by mixing the highly soluble precursor cisdiaminodihydroplatinum (II) with $\mathrm{KCl}$ [17]. In both cases, high drug entrapment efficiency was achieved with NP diameters of approximately $45 \mathrm{~nm}$ and $40 \mathrm{~nm}$ for GMP NP and Cisplatin NP, respectively.

Our current work aimed to develop a stroma-rich, subcutaneous xenograft bladder tumor model for evaluation of TAF-targeting anti-tumor drugs. We planned to use this model system to evaluate GMP NP and Cisplatin NP combination therapy (Combo NP) in terms of delivery, accumulation of drug within the tumor, and anti-tumor activity. We also set out to investigate the TAF-targeting capability of Combo NP with TAFs apoptosis, collagen deposition and changes in tumor permeability evaluated.

\section{Materials and Methods}

\subsection{Materials}

Gemcitabine monophosphate disodium salt (GMP, purity $297 \%$ ) was provided by Qualiber, Inc. (Chapel Hill, NC). Cisplatin (cis-diamminedichloroplatinum (II)) was purchased from Sigma-Aldrich (Dorset, UK). GMP NP (including ${ }^{3} \mathrm{H}$-labeled GMP NP) and Cisplatin NP were prepared as described previously [16,17]. Size distribution and zeta potential were measured on a Malvern ZetaSizer Nano series (Westborough, MA).

The human bladder transitional cell line UMUC3 was from the American Type Culture Collection (ATCC), and the mouse embryonic fibroblast cell line NIH 3T3 (UNC Tissue Culture Facility) were cultured in Dulbecco's modified Eagle's Media (DMEM) (Invitrogen, Carlsbad, CA) supplemented with penicillin $(100 \mathrm{U} / \mathrm{mL})$ and streptomycin $(100 \mu \mathrm{g} / \mathrm{mL})$ (Invitrogen), and 10\% fetal bovine serum (Sigma, St. Louis, MO)/10\% Bovine calf serum (Hyclone, Logan, Utah), respectively. Cells were cultivated in a humidified incubator at 37 ${ }^{\circ} \mathrm{C}$ and $5 \% \mathrm{CO}_{2}$ and harvested with $0.05 \%$ trypsin-EDTA before subculture.

Female nude mice $6-8$ weeks of age were used in all studies. All work performed on animals was approved by the Institutional Animal Care and Use Committee at University of North Carolina at Chapel Hill.

\subsection{Stroma-rich subcutaneous xenograft bladder tumor model}

To establish the xenograft model, UMUC3 $\left(5 \times 10^{6}\right)$ and NIH 3 T3 cells $\left(2 \times 10^{6}\right)$ in $100 \mu \mathrm{L}$ of PBS were subcutaneously co-injected with Matrigel (BD Biosciences, CA) at a ratio of 1:1 $(\mathrm{v} / \mathrm{v})$ into the right flank of mice. For the standard subcutaneous bladder tumor model, only UMUC3 cells $\left(5 \times 10^{6}\right)$ were injected. Tumor volume was measured every three days starting on day seven after inoculation. The formula: $\mathrm{V}=\left(\mathrm{L} \times \mathrm{W}^{2}\right) / 2$ was applied to calculate tumor volume, where $\mathrm{V}$ is the tumor volume, $\mathrm{L}$ the larger perpendicular diameter and $\mathrm{W}$ the 
smaller perpendicular diameter. Tumor growth was normalized to the original volume calculated on the first day of measurement. Tumor sections collected on day eight for the two animal models and also those of clinical patients (kindly supplied from Dr. William Kim, Lineberger Comprehensive Cancer Center, UNC) were stained with hematoxylin and eosin (H\&E), immunofluorescent staining for a-SMA (FITC labeled) and CD31 (Alexa Fluor 647 labeled). For immunofluorescence, slides were deparaffinized with xylene and a graded alcohol series. After antigen retrieval, sections were blocked with $10 \%$ goat serum and then incubated with polycolonal rabbit anti-a-SMA antibody (Abcam, Cambridge, MA, USA) and anti-CD31 (BD Biosciences, CA, USA) at 1:100 dilution overnight at $4{ }^{\circ} \mathrm{C}$. Immunocomplexes were visualized with the corresponding FITC-labeled and Alexa Fluor 647 secondary antibody at a 1:1000 and 1:100 dilution respectively for $1 \mathrm{~h}$ at room temperature in the dark. Slides were rinsed with PBS and cover-slipped with Vectashield containing DAPI (Vector laboratories, Burlingame, CA). Digital images were acquired by an Eclipse Ti-U inverted microscope (Nikon Corp., Tokyo, Japan) 20x magnification and quantitatively analyzed on Image $\mathbf{J}$ (National Institutes of Health).

\subsection{In vitro cell viability of free GMP and Cisplatin on UMUC3 cells and analysis of synergistic effects of free drug combinations}

MTT (3-[4,5-dimethylthiazol-2-yl]-2,5-diphenyltetrazolium bromide) assay was conducted to evaluate in vitro viability of free GMP, Cisplatin and their combinations. Briefly, cells were seeded in 96-well plates at a density of 3,000 cells per well $24 \mathrm{~h}$ prior to drug treatment. Subsequently, cells were treated with free drugs and drug combination with various molar ratio at a series of dilutions in full medium. Following $48 \mathrm{~h}$ treatment, $20 \mu \mathrm{L}$ MTT $(5 \mathrm{mg} / \mathrm{mL})$ reagent was added for an additional $4 \mathrm{~h}$ incubation at $37{ }^{\circ} \mathrm{C}$. The medium was discarded, the formed formazan salt was dissolved in $150 \mu \mathrm{L}$ of DMSO and absorbance was read at $570 \mathrm{~nm}$ using a multidetection microplate reader (Plate CHAMELEONTM VHidex). Cell survival rates were calculated as normalized to control untreated wells. Each concentration was tested in four wells and data presented in means \pm standard error means (SD). The mean drug concentration required for $50 \%$ growth inhibition $\left(\mathrm{IC}_{50}\right)$ was determined using CompuSyn software (Version 1.0, Combo-Syn Inc., U.S.) using the median effect equation: $\mathrm{Fa}=\left[1+\left(\mathrm{IC}_{50} / \mathrm{D}\right) \mathrm{m}\right]-1$, where $\mathrm{Fa}$ is the fraction of affected cells, $\mathrm{D}$ is drug concentration and $\mathrm{m}$ is the Hill slope.

Combination Index (CI) Analysis of free drug combination based on the Chou and Talalay method [18] was performed using CompuSyn software. Briefly, for each level of Fa the CI values for GMP and Cisplatin combinations were calculated according to the following equation: $\mathrm{CI}=(\mathrm{D}) 1 /(\mathrm{Dx}) 1+(\mathrm{D}) 2 /(\mathrm{Dx}) 2$, where (D) 1 and (D)2 are the concentrations of each drug in the combination resulting in Fa×100\% growth inhibition, and (Dx)1 and (Dx)2 are the concentrations of the drugs alone resulting in Fax100\% growth inhibition. CI values for drug combinations were plotted as a function of $\mathrm{Fa}$. CI values less than 1 or more than 1 demonstrate synergism or antagonism of drug combinations, respectively. The CI values between $\mathrm{Fa}=0.2$ and $\mathrm{Fa}=0.8$ are considered valid [19]. 


\subsection{Tumor accumulation of GMP and Cisplatin in established animal model}

To measure tumor accumulation of Combo Free and Combo NP, animals were randomly divided into two groups $(n=6)$ and intravenously injected with free GMP containing a tiny fraction of ${ }^{3} \mathrm{H}$-Labeled free cytidine monophosphate, which is believed to have the similar pharmacokinetic profile as GMP [16] and Cisplatin (Combo Free) and Combo NP at a dose of $16 \mathrm{mg} / \mathrm{kg}$ and $1.6 \mathrm{mg} / \mathrm{kg}$ respectively. Three mice from each group were sacrificed at each predestinate time point, and approximately $45 \mathrm{mg}$ of blood was withdrawn using the tail bleeding method. Tumor uptake of GMP and Cisplatin was expressed as the percentage of the injected dose per gram tumor. For measurement of GMP, 10 to $20 \mathrm{mg}$ of blood was immediately mixed with $10 \times \mathrm{NCS}^{\circledR}$ II Tissue Solubilizer (Amersham Biosciences, Inc) and digested at $60^{\circ} \mathrm{C}$ overnight. Three hundred $\mu \mathrm{L}$ of hydrogen peroxide (30\% in water, Fisher) was added to the samples and vortexed to bleach the blood color, and then the sample was mixed with $4 \mathrm{~mL}$ scintillation cocktail (Fisher Inc). The ${ }^{3} \mathrm{H}$ radioactivity in the blood samples was counted using a liquid scintillation analyzer (TRI-CARB 2900 TR, Packard Bioscience Co.). For the measurement of Cisplatin, approximately $30 \mathrm{mg}$ of blood was digested with $400 \mu \mathrm{L} 60 \%$ nitric acid (Acros Organic) at $70^{\circ} \mathrm{C}$ overnight and measured by Inductively Coupled Plasma-Mass Spectroscopy (ICP-MS, NexIONTM 300, Perkin Elmer Inc).

\subsection{Anti-tumor efficacy in stroma-rich xenograft bladder tumor model}

On day seven after implantation, mice were randomized by completely randomized design using a random number table into seven groups $(\mathrm{n}=5)$ as follows: Saline (Control group), free GMP (GMP Free), free Cisplatin (Cisplatin Free), combination of free GMP and Cisplatin (Combo Free), GMP NP, Cisplatin NP and combination of GMP NP and Cisplatin NP (Combo NP). For combination of free drugs or nanoparticles, free drugs or nanoparticles were mixed right before injection. Once they are mixed, the mixture was injected intravenously. IV injections were performed every three days for a total of 3 injections with the GMP dose at $16 \mathrm{mg} / \mathrm{kg}$ and Cisplatin at $1.6 \mathrm{mg} / \mathrm{kg}$. Tumor volume was measured every three days using the aforementioned method. Body weight was also recorded. Mice were sacrificed two days after the last injection by $\mathrm{CO}_{2}$ asphyxiation and tumors were excised. A portion of the tumor was fixed in 10\% formalin and paraffin-embedded sectioned for H\&E staining, TUNEL assay, and a-SMA immunofluorescence by an operator blinded to the treatment groups.

\subsection{In vivo toxicity analysis}

After three daily injections, blood was collected from the venous plexus of the eye and centrifuged at 4,000 rpm for $5 \mathrm{~min}$. Serum alanine aminotransferase (ALT), aspartate aminotransferase (AST), blood urea nitrogen (BUN) and creatinine levels were assayed as indicators of hepatic and renal function. Organs (heart, liver, spleen, lung, kidney) were fixed and sectioned for H\&E staining.

\subsection{Tissue analysis}

Tumor sections were stained for TUNEL assays as recommended by the manufacturer (Promega, Madison, WI). DAPI mounting medium was dropped on the sections for nuclear 
staining. Apoptosis was also determined by H\&E staining. Tumors were also prepared for a-SMA immunofluorescence (FITC label). Images were acquired by an Eclipse Ti-U inverted microscope (Nikon Corp., Tokyo, Japan) at 20× magnification and analyzed on Image J (National Institutes of Health).

\subsection{TAF-targeting efficacy in stroma-rich xenograft bladder tumor model}

Mice with $200 \mathrm{~mm}^{3}$ tumors were treated with a single dose of Combo NP (GMP $16 \mathrm{mg} / \mathrm{kg}$, Cisplatin $1.6 \mathrm{mg} / \mathrm{kg})(\mathrm{n}=24)$. Every 24 hours, mice were sacrificed. Tumors were excised and sectioned for a-SMA (Alaxa Fluor 647)/TUNEL immunofluorescence double staining and Masson's trichrome (Sigma, USA).

\subsection{Analysis of tumor permeability}

Drug permeability in the tumor was evaluated by Evans Blue assay [20]. Tumor-bearing mice were randomized into three groups $(n=3)$ as follows: Control group, Combo Free and Combo NP. Single intravenous injection was performed for each treatment with the GMP dose at $16 \mathrm{mg} / \mathrm{kg}$ and Cisplatin at $1.6 \mathrm{mg} / \mathrm{kg}$. Twenty-four hours later, $100 \mathrm{mg} / \mathrm{kg}$ of Evans Blue $(10 \mathrm{mg} / \mathrm{ml}$ in $0.9 \% \mathrm{NaCl})$ was intravenously injected. After $30 \mathrm{~min}$, mice were sacrificed and blood and tumor tissue were homogenized in a $0.1 \%$ sodium sulfate/acetone mixture $(7: 3 \mathrm{v} / \mathrm{v})$ at a ratio of 1:9. Samples were maintained at ambient temperature in the dark overnight and then centrifuged at 1,000 g for $5 \mathrm{~min}$. Supernatants were used to determine absorbance at $620 \mathrm{~nm}$. The amount of Evans Blue in tumor tissue was expressed as $\mu \mathrm{g} / \mathrm{g}$ tissue.

\subsection{Statistical analysis}

Results were expressed as a mean \pm standard deviation (S.D.). Student's t-test and one-way analysis of variance (ANOVA) test were used to evaluate statistical significance. A p value of $p<0.05$ was considered to indicate statistical significance.

\section{Results and discussion}

\subsection{Characterization of GMP NP and Cisplatin NP}

The dioleoylphosphatydic acid (DOPA) was employed as a pre-coating reagent for the nanosized $\mathrm{CaP}$ cores. The lipid coating prevents the core from aggregation during the centrifugal separation step and makes it soluble in chloroform. The DOPA layer coating CaP core served as the inner leaflet lipid and a variety of lipids for the outer leaflet could simply be added into the $\mathrm{CaP}$ core solution in chloroform. The choice of the asymmetric outer lipids plays an important role in the pharmacokinetics and tissue distribution of the final nanoparticles [21]. Asymmetric lipid bilayer-modified nanoparticles for GMP and Cisplatin were prepared as previously reported [16,17]. Particles with a core-shell shape were $44.5 \pm 0.2 \mathrm{~nm}$ and $40.6 \pm 0.2 \mathrm{~nm}$ for GMP NP and Cisplatin NP, respectively. Particles exhibited high entrapment efficiency at around $49.5 \%$ and $44 \%$, and zeta potential of $11.5 \pm 0.6 \mathrm{mV}$ and $5.6 \pm 0.3 \mathrm{mV}$ for GMP NP and Cisplatin NP, respectively. The drug loading was $23 \pm 2 \mathrm{wt} \%(\mathrm{n}=3)$ and $82 \pm 5 \mathrm{wt} \%(\mathrm{n}=3)$ for GMP NP and Cisplatin NP, respectively (Fig. S1). 


\subsection{Histopathology for stroma-rich subcutaneous xenograft bladder tumor model}

Xenografts with UMUC3 and/or NIH 3T3 cells and patient tumor sections were stained with H\&E and for a-SMA (FITC labeled). Our tumor model was enriched with stroma structure (H\&E) and a-SMA positive cells (17.5 $\pm 3.2 \%$, UMUC3/NIH 3T3) compared to the common subcutaneous bladder model with injection of UMUC3 cells alone (1.8 $\pm 0.6 \%$, UMUC3), and more closely resembles human bladder tumor structure (14.8 $2.1 \%$, Patient) (Fig. 1A). Alpha-SMA-positive fibroblasts co-located with CD31-positive blood vessel endothelial cells in our established tumor model (Fig. 1B). The stroma-rich tumor (co-injection with UMUC3 and NIH 3T3 cells) grew more rapidly (Fig. 1C).

No tumor growth was observed if only NIH 3T3 cells were inoculated. Therefore, such a stroma-rich tumor closely mimics the structure of human bladder cancer isolates, which should enable us to better understand the synergistic effect of Combo NP on tumor inhibition and TAF-targeting effect.

\subsection{Synergistic effect induced by tumor accumulation of GMP NP and Cisplatin NP}

Gemcitabine has been reported to affect the expression of key proteins involved in nucleotide excision repair (NER) and mismatch repair (MMR), thereby inhibiting repair of DNA damage caused by Cisplatin [22]. Synergy between gemcitabine and Cisplatin might be related to the reduced DNA repair of intra-strand and possibly inter-strand cross-links of Cisplatin [23]. In vitro toxicity analysis of combined GMP and Cisplatin was performed after $48 \mathrm{~h}$ treatment of UMUC3 cells (Fig. 2). The combination index (CI) was calculated using a series of molar ratios and amounts of GMP and Cisplatin. CI values were analyzed according to Chou and Talalay [18] using CompuSyn software (ComboSyn, Inc., Paramus, NJ, USA). The CI fell between 0.1 and 0.3 over a broad range of drug ratios from the CI vs Fa plot (Fig. 2B). Synergy, as indicated by a CI $<1$, therefore, is assumed to be induced over a wide range of free drug ratios. Within the range of drug ratios, the synergistic effect of GMP and Cisplatin at a molar ratio of 10 to 1 , not only as free drugs but also in nanoparticles, was further affirmed by in vitro cytotoxicity study of GMP NP, Cisplatin NP, and Combo NP (Fig. S2). The combination of GMP NP and Cisplatin NP (molar ratio of 10:1) was indicated to exhibit better performance than single nanoparticles alone over a range of concentrations with the lowest $\mathrm{IC}_{50}$ and small $\mathrm{CI}$ value under one. According to the in vitro data for free drugs and nanoparticles, we apply the combination of GMP NP and Cisplatin NP at synergistic ratio for further in vivo experiments.

Next, we investigated the synergistic effect of Combo NP and Combo Free in vivo. The resulting pharmacokinetics (Fig. S3) suggests that Combo NP provides advantages over Combo Free. About 40 to $45 \%$ of the GMP NP were retained in the blood circulation $2 \mathrm{~h}$ after bolus administration, while the free GMP was rapidly cleared within 20 min. AUC and half-life of Cisplatin NP were 1.4 to 1.7 times higher than that of Cisplatin Free, for which more than $80 \%$ had been cleared after the first 20 min. Incorporation of GMP and Cisplatin in lipid bilayer coated nanoparticles modified with hydrophilic polyethylene glycol increased the drug retention in the circulation and reduced the drug clearance which is consistent with our previous pharmacokinetic studies [16]. However, there is significant discrepancy in the PK profile of GMP NP and Cisplatin NP. One explanation for the 
difference is that GMP NP with highly positive charges $(11.5 \mathrm{mV})$ are more likely to bind with serum proteins, leading to longer circulation in the system but lower tissue distribution; however, Cisplatin NP with relatively neutral charges $(5.6 \mathrm{mV})$ worked in the opposite way. Moreover, Cisplatin NP produced a slightly higher accumulation in tumor than GMP NP after a single injection. Both nanoparticles showed significantly higher tumor accumulation (more than $10 \%$ of injected dose per gram remained in the tumor $10 \mathrm{~h}$ post injection) than the free drugs (less than $2 \%$ of injected dose per gram left $10 \mathrm{~h}$ post injection) (Fig. 2C). One possible reason is the phenomenon known as enhanced permeability and retention (EPR). The vasculature of tumors is comprised of poorly aligned and defective endothelial cells lacking innervation [24,25]. Nanoparticle formulations can increase tumor accumulation through the EPR effect and with a specific targeting ligand can enhance tumor cell uptake through a receptor mediated pathway.

The ratio of GMP to Cisplatin (mol: mol) in tumor after Combo NP injection, which is in the wide synergistic range of free drugs (Fig. 2B) was 8.35:1, and 6.75:1 at $5 \mathrm{~h}$ and $10 \mathrm{~h}$ after injection (Fig. 2C); therefore, a synergistic effect took place. When taken together and incorporated into nanoparticles, GMP and Cisplatin exhibited increased drug retention in the circulation and enhanced tumor accumulation without altering the synergy. This may result in more potent anti-tumor effects and reduced side effects [26,27].

\subsection{Anti-tumor effect of combined therapy}

Here, we report the potent anti-tumor efficacy of the Combo NP in our stroma-rich bladder tumor model at a dose of $16 \mathrm{mg} / \mathrm{kg}$ for GMP and $1.6 \mathrm{mg} / \mathrm{kg}$ for Cisplatin. We first determined whether Combo NP can exhibit synergistic anti-tumor effects without significant adverse effects (Fig. 3).

When treatment began on day seven after inoculation, free Cisplatin $(1.6 \mathrm{mg} / \mathrm{kg})$ monotherapy showed little anti-tumor effect, with Cisplatin NP being more effective than free drug $(\mathrm{p}<0.05)$. Tumor growth was delayed significantly in mice treated with free GMP and GMP NP compared to the control group after multiple doses. In comparison, Combo NP treated tumors had significantly smaller volume than the other six groups at the end of the experiment, showing growth of only 0.3 fold more than the day one tumor volume. No weight loss was observed in any treatment group, indicating that the treatment was well tolerated (data not shown). The enhanced tumor growth inhibition exhibited by nanoparticles should be attributed to the endocytosis mediated by the sigma receptors and EPR effect [28], which is also indicated by the tumor accumulation study aforementioned. Nanoparticle formulation with specific targeting ligand can increase tumor accumulation through EPR effect and enhanced tumor cell uptake through receptor-mediated pathway. Furthermore, in vivo maintenance of drug ratios shown to be synergistic in vitro was proven to provide increased efficacy in preclinical tumor models [29]. Therefore, the synergistic effect proven by the tumor accumulation study (Fig. 2), enabled the Combo NP to show the most outstanding effect on tumor growth inhibition in comparison with GMP NP and Cisplatin NP alone. 


\subsection{In vivo toxicity}

To test the toxicity of combined GMP NP and Cisplatin NP, blood was obtained for hematological analysis and histopathology of organs was evaluated by H\&E staining. Gemcitabine and Cisplatin are notable for their potential hepatic and renal toxicities respectively. In our toxicity studies, AST increased slightly in Combo NP group, but remained within the normal range (Table 1) [16]. No noticeable histological changes were seen in H\&E-stained tissue sections of heart, liver, spleen, lung and kidney (Fig. S4). There were no noticeable changes between the control and Combo NP group, which showed no evidence of organ toxicity. These findings suggest the potential application of nanoparticles in clinic.

\subsection{Combo NP triggers cell apoptosis and inhibits fibroblasts growth}

Stroma-rich tumor-bearing mice were sacrificed two days after the final injection and the tumors were sectioned for TUNEL assay and a-SMA immunohistochemistry. In the TUNEL assay (Fig. 4A), the Combo NP exhibited the most effective killing effects and induced a 16.1 fold higher amount of apoptotic cells compared with the control group. This was more potent than the Combo Free treatment group, which showed $55.5 \%$ less apoptosis than that of the Combo NP. There was no significant difference among Combo Free, GMP NP and Cisplatin NP groups ( $>0.05$ ). Cisplatin Free treatment had limited ability to induce apoptosis in tumor cells. The results indicate the combination of GMP NP and Cisplatin NP augments the intrinsic cytotoxicity. This finding is consistent with data in Figure 3 that the Combo NP exerts synergistic anti-tumor effects.

Meanwhile, Combo NP not only elicited an apoptosis-inducing effect on carcinoma cells, but also changed the number of a-SMA-positive fibroblasts in the tumor extracellular matrix. To assay for this change, we used immunofluorescence to measure the percentage of a-SMA-positive fibroblasts in the normal untreated tumor and in tumors treated with different preparations (Fig. 4). a-SMA was significantly decreased in the Combo NP group compared to the untreated group $(0.7 \pm 0.3 \%$ vs. $17.6 \pm 3.2 \%$, percentage of positive-stained cells, $\mathrm{p}<0.05)$. The fibroblasts expression after Combo NP treatment was $89.7 \%, 87.2 \%$ and 94.3\% lower than that after GMP Combo NP 58.3 $\pm 6.8252 \pm 10.35 .3 \pm 0.40 .2 \pm 0.1 \mathrm{NP}$, Cisplatin NP, and Combo Free treatment, respectively $(\mathrm{p}<0.05)$. Those results suggest the importance of the combination of GMP NP and Cisplatin NP for in vivo efficacy. H\&E staining also indicated that the Combo NP caused the most severe necrosis in the tumor.

\subsection{Combo NP depletes TAFs in the tumor, modifies collagen deposition}

Taking advantage of the stroma-rich animal model, the interaction of TAFs and the combined drugs was also investigated. The effect of a single Combo NP injection treatment on fibroblasts and tumor cells in the stroma-rich bladder tumor model was investigated from day one to day seven after treatment. Day 0 before treatment was also investigated.

Fig. 5A-C present the double staining for TUNEL and a-SMA and the quantitative results. Firstly, $4.9 \%$ of cells were induced to undergo apoptosis one day after Combo NP injection, among which 56.6\% apoptotic cells were a-SMA-positive. This suggests that the fibroblasts have a greater tendency to be killed compared to tumor cells. From day one to day two, the

J Control Release. Author manuscript; available in PMC 2015 May 28. 
apoptotic fibroblasts in total apoptotic cells increased dramatically resulting in more than $60 \%$ apoptotic fibroblasts. The apoptotic cells in fibroblasts reached $87.2 \%$ on day four, which was $45 \%$ higher than that in carcinoma cells. The results indicate that the apoptosis of fibroblasts not only began before that of carcinoma cells but also substantially resulted in fibroblast depletion. By the last two days, the killing effect of the Combo NP was reduced and fewer cells were apoptotic, with the increasing number of a-SMA-positive fibroblasts. Therefore, the apoptotic fibroblast (\%) in total fibroblast was significantly decreased to nearly $1 \%$. Secondly, the collagen on day four became $14.9 \%$ of the original collagen present on day 0 , a value also different from that of day three. This shows that the killing effect of Combo NP on TAFs in the first three days resulted in the decreased deposition of collagen on day four. Collage deposition showed an increasing trend starting on day five (Fig. 5D).

As it is shown in Fig. 4, Combo Free, GMP NP, and Cisplatin NP exhibited partial efficacy in reducing fibroblasts, but Combo NP showed the fewest a-SMA positive cells and induced the most tumor cell apoptosis compared to all the treatment groups $(\mathrm{p}<0.05)$. The data suggest that the combination therapy may have a TAF-targeted effect by inducing cell apoptosis with a strong inhibition of stroma cell proliferation. Under that hypothesis, we administered a single injection of Combo NP and detected the apoptotic fibroblasts every 24 $\mathrm{h}$ after injection. The larger amount of apoptotic fibroblasts compared to apoptotic tumor cells and collagen re-deposition indicated that tumor stroma was the target for the Combo NP and tumor cell killing could be the secondary role of the Combo NP. The TAF-targeting effect could be interpreted by the following two explanations: (1) the myofibroblasts were much more sensitive to the Combo NP than that of bladder carcinoma cells. (2) Tumor blood vessels are reported to be located in the stroma [30,31] and Fig. 1B also indicates that some CD31-positive cells are co-located with a-SMA-positive fibroblasts. Our explanation could be that Combo NP, once delivered through tumor vessels and transferred from the vessel wall with high permeability through extra cellular matrix to the tumor cells will exhibit killing effect on the TAFs as the first target instead of tumor cells since the TAFs are distributed along the vessel or near the vessel.

\subsection{Tumor vessel permeability}

Passive targeting of liposomal formulations is mediated mainly by the enhanced vascular permeability of tumor vessels. To evaluate vascular permeability after Combo Free and Combo NP treatment, tumor bearing mice were intravenously injected with Evans Blue, which binds to serum albumin and tends to accumulate in tissues with leaky vasculature [20]. Vascular permeability was found to be nearly 21.3 and 2.7 times higher in the Combo NP group than in the Control and Combo Free groups, respectively (Fig. 6). Besides the increasing transport through vasculature, the enhanced intratumoral uptake of Evans Blue might also be contributed to tumor priming effect by substantial apoptosis caused by pretreatment with single injection of Combo NP (Fig. 5). This could reduce tumor cell density, expend the interstitial space and then promote the penetration into threedimensional tumor histocultures $[32,33]$. Further investigation is desired for the mechanism of greater tumor uptake of Evans Blue. 
The more pronounced accumulation from Combo NP could be attributed to the synergistic effect of GMP NP and Cisplatin NP on the tumor blood vessels: (1) Combo NP could significantly reduce the expression of vascular endothelial growth factor (VEGF) (Fig. S5), which is an important mediator of tumor angiogenesis, and enhance the extent of tumor vasculature abnormalities, which impairs the tumor growth [34-36]. This finding is consistent with previous result that Combo NP exerts higher tumor inhibition (Fig. 3); (2) Fig. 5 indicates that because of their sensitivity or proximity to tumor blood vessels, the TAFs will be first killed by nanoparticles distributed in the stoma and the collagen in stroma will be depleted. This depleting effect could be helpful for better distribution of antitumor compounds in the tumor area which would not otherwise access the tumor cells [37], and enhance the total drug tumor accumulation and transport to carcinoma cells.

\section{Conclusions}

In this study, anti-tumor effects of Combo NP were investigated in a stroma-rich xenograft bladder cancer model. GMP and Cisplatin accumulated in treated tumor tissue at a molar ratio and concentration expected to have synergistic anti-tumor effects. Combo NP's ability to inhibit tumor growth was enhanced relative to Combo Free with no obvious toxicity. The effectiveness of Combo NP could be attributed to its effect on both carcinoma cells and TAFs. The anti-tumor activity of Combo NP may result from the combined effects of TAFs depletion with alterations in collagen deposition and increased uptake of chemotherapeutic drugs by the tumor and apoptosis of carcinoma cells. Since the standard first line chemotherapy for muscle invasive bladder cancer is now gemcitabine plus Cisplatin $[38,39]$, the result of our study suggest that Combo NPs could be tested clinically to replace free drug combination.

\section{Acknowledgments}

This project was supported by NIH grants CA129835, CA129421, CA151652, CA151455 and CA149363. Dr. Jing Zhang's work in UNC was supported by the National Natural Science Foundation of China (NO. 81202927). We thank Steven Plonk and Andrew Blair for editing the manuscript.

\section{Abbreviations}

a-SMA

AST

ALT

BUN

GMP

Cisplatin NP

Combo Free

Combo NP

EPR
a-Smooth muscle actin

Aspartate aminotransferase

Alanine aminotransferase

Blood urine nitrogen

Gemcitabine monophosphate

Cisplatin nanoparitcles

Combination of GMP and Cisplatin free drugs

Combination of GMP NP and Cisplatin NP

Enhanced permeability and retention 


$\begin{array}{ll}\text { GMP NP } & \text { Gemcitabine monophosphate nanoparticles } \\ \text { H\&E } & \text { Hematoxylin and eosin } \\ \text { TAFs } & \text { Tumor-associated fibroblasts } \\ \text { TUNEL } & \text { TdT-mediated dUTP Nick-End Labeling } \\ \text { VEGF } & \text { Vascular endothelial growth factor }\end{array}$

\section{Reference}

1. Grasso M. Bladder cancer: a major public health issue. Eur. Urol. 2008; 7:510-515.

2. Sylvester RJ, van der Meijden AP, Oosterlinck W, Witjes JA, Bouffioux C, Denis L, Newling DW, Kurth K. Predicting recurrence and progression in individual patients with stage Ta T1 bladder cancer using EORTC risk tables: a combined analysis of 2596 patients from seven EORTC trials. Eur. Urol. 2006; 49:466-475. [PubMed: 16442208]

3. Parada B, Reis F, Cerejo R, Garrido P, Sereno J, Xavier-Cunha M, Neto P, Mota A, Figueiredo A, Teixeira F. Omega-3 fatty acids inhibit tumor growth in a rat model of bladder cancer. Biomed. Res. Int. 2013

4. Cosco D, Paolino D, Cilurzo F, Casale F, Fresta M. Gemcitabine and tamoxifen-loaded liposomes as multidrug carriers for the treatment of breast cancer diseases. Int. J. Pharm. 2012; 17:229-237. [PubMed: 22093954]

5. Gabizon AA, Shmeeda H, Zalipsky S. Pros and cons of the liposome platform in cancer drug targeting. J. Liposome Res. 2006; 16:175-183. [PubMed: 16952872]

6. Matsumura Y, Maeda H. A new concept for macromolecular therapeutics in cancer chemotherapy: mechanisms of tumoritropic accumulation of proteins and the antitumor agent smancs. Cancer Res. 1986; 46:6387-6392. [PubMed: 2946403]

7. Albini A, Sporn MB. The tumour microenvironment as a target for chemoprevention. Nat. Rev. Cancer. 2007; 7:139-147. [PubMed: 17218951]

8. Zhang J, Liu JS. Tumor stroma as targets for cancer therapy. Pharmcol. Ther. 2013; 137:200-215.

9. Pietras K, Östman A. Hall marks of cancer: Interactions with the tumor stroma. Experimental Cell Res. 2010; 316:1324-1331.

10. Mueller MM. Fusenig NE. Friends or foes-bipolar effects of the tumour stroma in cancer. Nat. Rev. Cancer. 2004; 4:839-849. [PubMed: 15516957]

11. Olive KP, Jacobetz MA, Davidson CJ, Gopinathan A, Mclntyre D, Honess D, Madhu B, Goldgraben MA, Caldwell ME, Allard D, Frese KK, Denicola G, Feig C, Combs C, Winter SP, Ireland-Zecchini H, Reichelt S, Howat WJ, Chang A, Dhara M, Wang L, Rückert F, Grützmann R, Pilarsky C, Izeradjene K, Hingorani SR, Huang P, Davies SE, Plunkett W, Egorin M, Hruban RH, Whitebread N, McGovern K, Adams J, Iacobuzio-Donahue C, Griffiths J, Tuveson DA. Inhibition of hedgehog signaling enhances delivery of chemotherapy in a mouse model of pancreatic cancer. Science. 2009; 324:1457-1461. [PubMed: 19460966]

12. Gonda TA, Varro A, Wang TC, Tycko B. Molecular biology of cancer-associated fibroblasts: can these cells be targeted in anti-cancer therapy? Semin. Cell Dev. Biol. 2010; 21:2-10. [PubMed: 19840860]

13. Alexa A, Baderca F, Lighezan R, Izvernariu D. Myofibroblasts reaction in urothelial carcinomas. Rom. J. Morphol. Embryol. 2009; 50:639-643. [PubMed: 19942959]

14. Jacob D, Davis J, Fang BL. Xenografitic tumor models in mice for cancer research, a technical review. Gene Ther. Mol. Biol. 2004; 18:213-219.

15. Hurst RE, Hauser PJ, Kyker KD, Heinlen JE, Hodde JP, Hiles MC, Kosanke SD, Dozmorov M, Ihnat MA. Suppression and activation of the malignant phenotype by extracellular matrix in xenograft models of bladder cancer: a model for tumor cell "dormancy". Plos One. 2013; 8:1-10. 
16. Zhang Y, Schwerbrock NM, Rogers AB, Kim WY, Huang L. Codelivery of VEGF siRNA and gemcitabine monophosphate in a single nanoparticle formulation for effective treatment of NSCLC. Mol. Ther. 2013; 21:1559-1569. [PubMed: 23774791]

17. Guo ST, Wang YH, Miao L, Xu ZH, Lin CM, Zhang Y, Huang L. Lipid-coated cisplatin nanoparticles induce neighboring effect and exhibit enhanced anticancer efficacy. ACS Nano. 2013; 7:9896-9904. [PubMed: 24083505]

18. Chou TC, Talalay P. Quantitative analysis of dose-effect relationships: the combined effects of multiple drugs or enzyme inhibitors. Adv. Enzyme Regul. 1984; 22:27-55. [PubMed: 6382953]

19. Han Y, He Z, Schulz A, Bronich TK, Jordan R, Luxenhofer R, Kabanov AV. Synergistic Combinations of Multiple Chemotherapeutic Agents in High Capacity Poly(2-oxazoline) Micelles. Mol. Pharm. 2012; 9:2302-2313. [PubMed: 22681126]

20. Bornmann C, Graeser R, Esser N, Ziroli V, Jantscheff P, Keck T, Unger C, Hopt UT, Adam U, Schaechtele C, Massing U, von Dobschuetz E. A new liposomal formulation of Gemcitabine is active in an orthotopic mouse model of pancreatic cancer accessible to bioluminescence imaging. Cancer Chemother. Pharmacol. 2008; 61:395-405. [PubMed: 17554540]

21. Li J, Yang Y, Huang L. Calcium phosphate nanoparticles with an asymmetric lipid bilayer coating for siRNA delivery to the tumor. J. Control. Release. 2012; 158:108-114. [PubMed: 22056915]

22. Peters GJ, Van Moorsel CJ, Lakerveld B, Smid K, Noordhuis P, Comijn EC, Weaver D, Willey JC, Voorn D, Van der Vijgh WJ, Pinedo HM. Effects of gemcitabine on cis-platinum-DNA adduct formation and repair in a panel of gemcitabine and cisplatin-sensitive or -resistant human ovarian cancer cell lines. Int. J. Oncol. 2006; 28:237-244. [PubMed: 16328001]

23. Besançon OG, Tytgat GA, Meinsma R, Leen R, Hoebink J, Kalayda GV, Jaehde U, Caron HN, van Kuilenburg AB. Synergistic interaction between cisplatin and gemcitabine in neuroblastoma cell lines and multicellular tumor spheroids. Cancer Lett. 2012; 319:23-30. [PubMed: 22182450]

24. Walsh MD, Hanna SK, Sen J, Rawal S, Cabral CB, Yurkovetskiy AV, Fram RJ, Lowinger TB, Zamboni WC. Pharmacokinetics and antitumor efficacy of XMT-1001, a novel, polymeric topoisomerase I inhibitor, in mice bearing HT-29 human colon carcinoma xenografts. Clin. Cancer Res. 2012; 18:2591-2602. [PubMed: 22392910]

25. Greish K. Enhanced permeability and retention of macromolecular drugs in solid tumors: a royal gate for targeted anticancer nanomedicines. J. Drug Target. 2007; 15:457-464. [PubMed: 17671892]

26. Li SD, Huang L. Nanoparticles evading the reticuloendothelial system: role of the supported bilayer. Biochim. Biophys. Acta. 2009; 1788:2259-2266. [PubMed: 19595666]

27. Matsumura Y, Maeda H. A new concept for macromolecular therapeutics in cancer chemotherapy: mechanism of tumoritropic accumulation of proteins and the antitumor agent smancs. Cancer Res. 1986; 46:6387-6392. [PubMed: 2946403]

28. Banerjee R, Tyagi P, Li S, Huang L. Anisamide-targeted stealth liposomes: a potent carrier for targeting doxorubicin to human prostate cancer cells. Int. J. Cancer. 2004; 112:693-700. [PubMed: 15382053]

29. Mayer LD, Harasym TO, Tardi PG, Harasym NL, Shew CR, Johnstone SA, Ramsay EC, Bally MB, Janoff AS. Ratiometric dosing of anticancer drug combinations: controlling drug ratios after systemic administration regulates therapeutic activity in tumor-bearing mice. Mol. Cancer Ther. 2006; 5:1854-1863. [PubMed: 16891472]

30. Liu J, Liao S, Diop-Frimpong B, Chen W, Goel S, Naxerova K, Ancukiewicz M, Boucher Y, Jain RK, Xu L. TGF- $\beta$ blockade improves the distribution and efficacy of therapeutics in breast carcinoma by normalizing the tumor stroma. Proc. Natl. Acad. Sci. USA. 2012; 109:16618-16623. [PubMed: 22996328]

31. Shimoda M, Mellody KT, Orimo A. Carcinoma-associated fibroblasts are a rate-limiting determinant for tumour progression. Semin Cell Dev. Biol. 2010; 21:19-25. [PubMed: 19857592]

32. Jang SH, Wientjes MG, Au JL. Enhancement of paclitaxel delivery to solid tumors by apoptosisinducing pretreatment: effect of treatment schedule. J. Pharmacol. Exp. Ther. 2001; 296:10351042. [PubMed: 11181938]

33. Lu D, Wientjes MG, Lu Z, Au JL. Tumor priming enhances delivery and efficacy of nanomedicines. J. Pharmacol. Exp. Ther. 2007; 322:80-88. [PubMed: 17420296] 
34. Kim KJ, Li B, Winer J, Armanini M, Gillett N, Phillips HS, Ferrara N. Inhibition of vascular endothelial growth factor-induced angiogenesis suppresses tumour growth in vivo. Nature. 1993; 362:841-844. [PubMed: 7683111]

35. Kuwahara K, Sasaki T, Kobayashi K, Noma B, Serikawa M, Liboshi T, Miyata H, Kuwada Y, Murakami M, Yamasaki S, Kariya K, Morinaka K, Chayama K. Gemcitabine suppresses malignant ascites of human pancreatic cancer: correlation with VEGF expression in ascites. Oncol. Rep. 2004; 11:73-80. [PubMed: 14654905]

36. Ikeda R, Vermeulen LC, Jiang Z, Lau E, Koledar JM. Gemcitabine and paclitaxel suppress the production of vascular endothelial growth factor induced by deferoxamine in human non-small cell lung cancer A549 cells. Exp. Ther. Med. 2010; 1:853-857. [PubMed: 22993609]

37. Dimou A, Syrigos KN, Saif MW. Overcoming the stromal barrier: technologies to optimized drug delivery in pancreatic cancer. Ther. Adv. Med. Oncol. 2012; 4:271-279. [PubMed: 22942909]

38. NCCN Clinical Practice Guidelines in Oncology: Bladder Cancer. v.1. 2013

39. Dash A, Pettus JA 4th, Herr HW, Bochner BH, Dalbagni G, Donat SM, Russo P, Boyle MG, Milowsky MI, Bajorin DF. A role for neoadjuvant gemcitabine plus cisplatin in muscle-invasive urothelial carcinoma of the bladder: a retrospective experience. Cancer. 2008; 113:2471-2477. [PubMed: 18823036] 
A

B
C

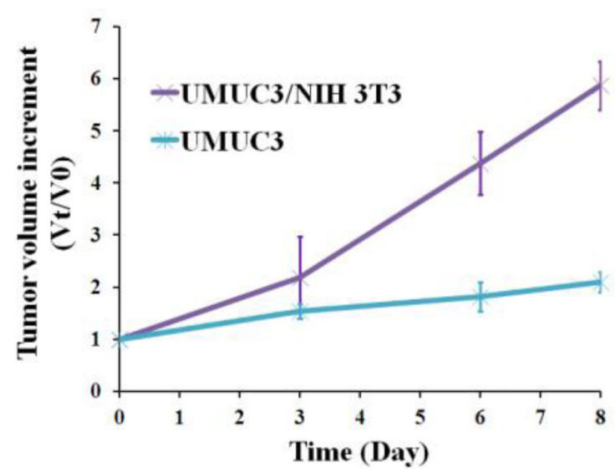

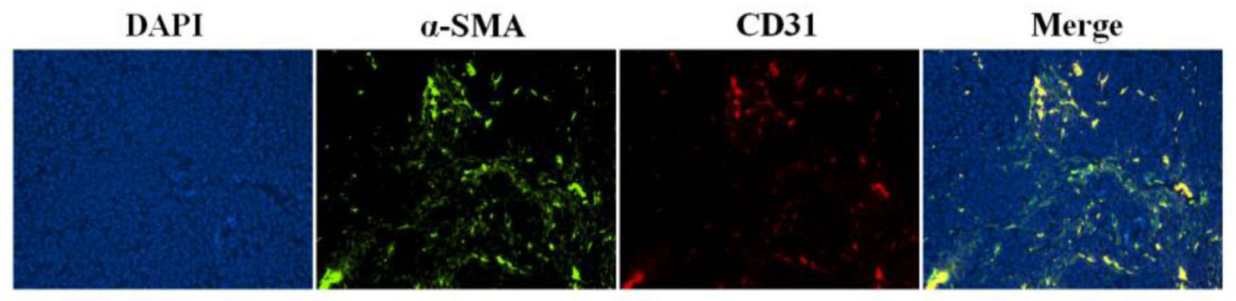

Fig. 1.

Histopathology for tumor formed by injection of UMUC3 cells (20x) without or with NIH 3T3 cells and from patient section (A); the expression of a-SMA, CD31 and merge (a-SMA: green; CD31: red; co-located: yellow) (B) in tumor formed by coinjection of UMUC3 with NIH 3T3 cells; tumor growth curves of different inoculations were charted since the tumor volume reached $200 \mathrm{~mm}^{3}(\mathrm{C})$. Tumor growth increment was presented by normalizing to the original volume on the $1^{\text {st }}$ day of measurement $(\mathrm{Vt} / \mathrm{V} 0)(\mathrm{n}=5)$. 

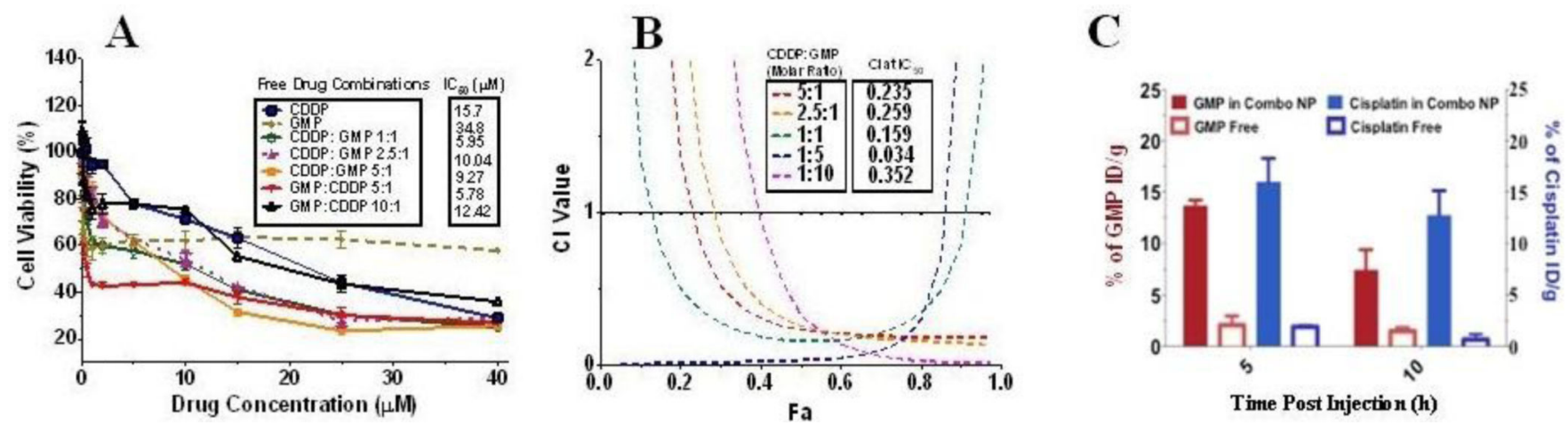

Fig. 2.

Cytotoxicity study of free GMP, Cisplatin and Combo Free at variable molar ratios (A) with the corresponding CI vs Fa plot (B), and tumor accumulation of GMP NP and GMP Free (Red bars), and Cisplatin NP and Cisplatin Free (Blue bars) at $5 \mathrm{~h}$ and $10 \mathrm{~h}$ after administration $(\mathrm{n}=3)(\mathrm{C})$. 


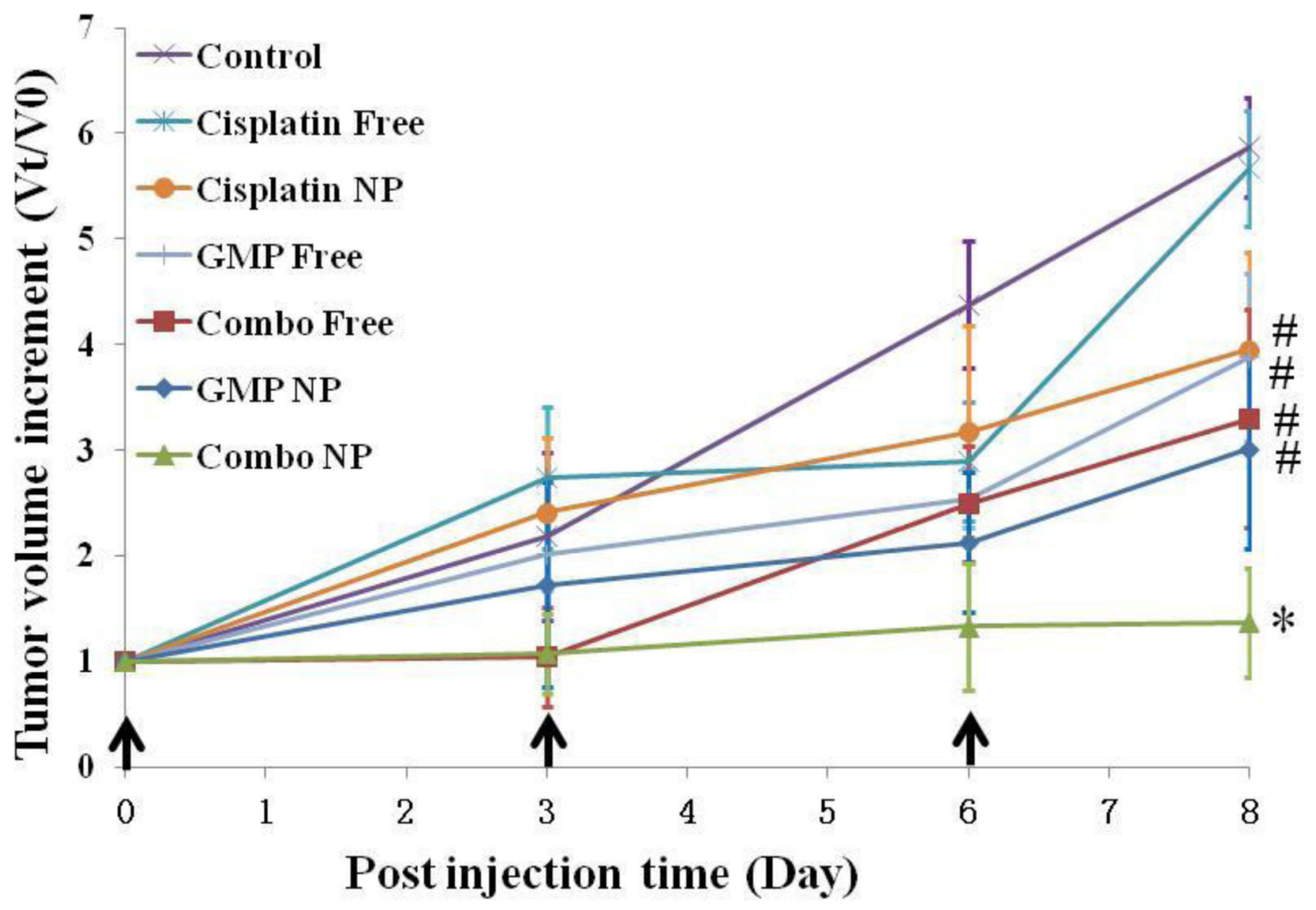

Fig. 3.

Tumor growth inhibition effects of different formulations on stroma-rich tumor bearing mice. GMP Free, Cisplatin Free, Combo Free, GMP NP, Cisplatin NP and Combo NP were administered intravenously every third day for a total of three injections as indicated by arrows. Data are mean \pm S.D. Statistics are as follows: ${ }^{*} \mathrm{p}<0.05$ vs. Control; \#p<0.05 vs. Combo NP; there is no significant difference among groups marked with “\#”, n=5. 
A
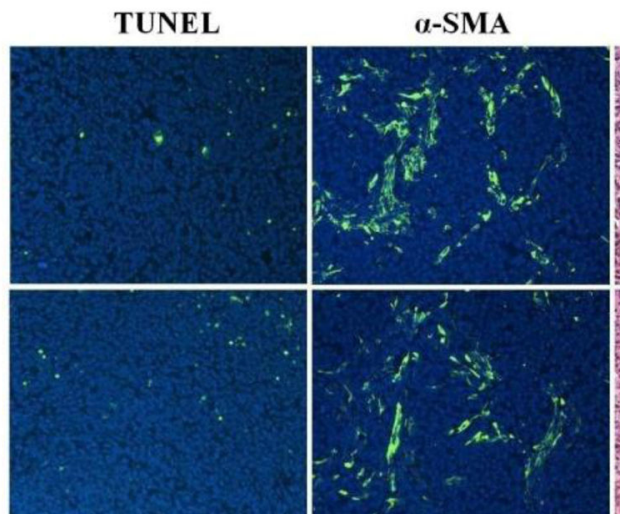

GMP Free

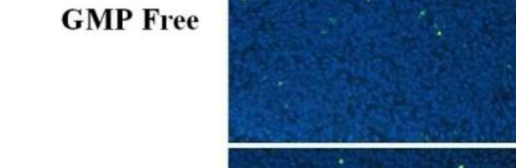

Cisplatin Free
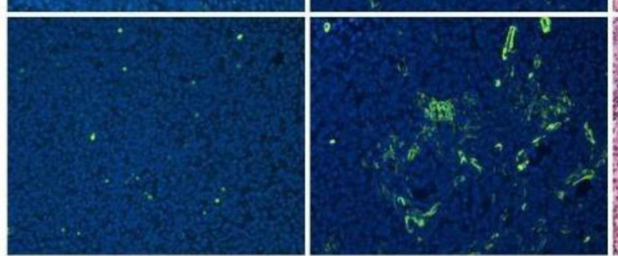

Combo Free
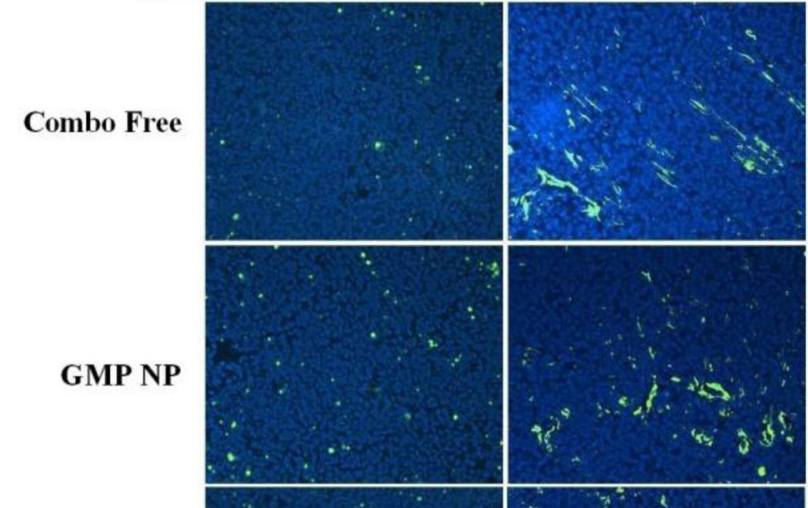

Cisplatin NP
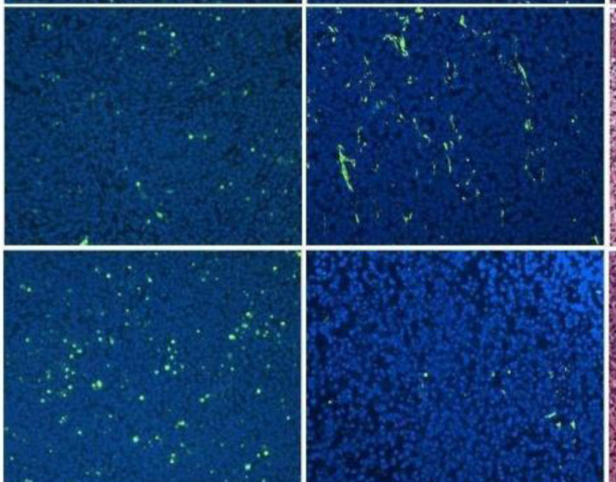
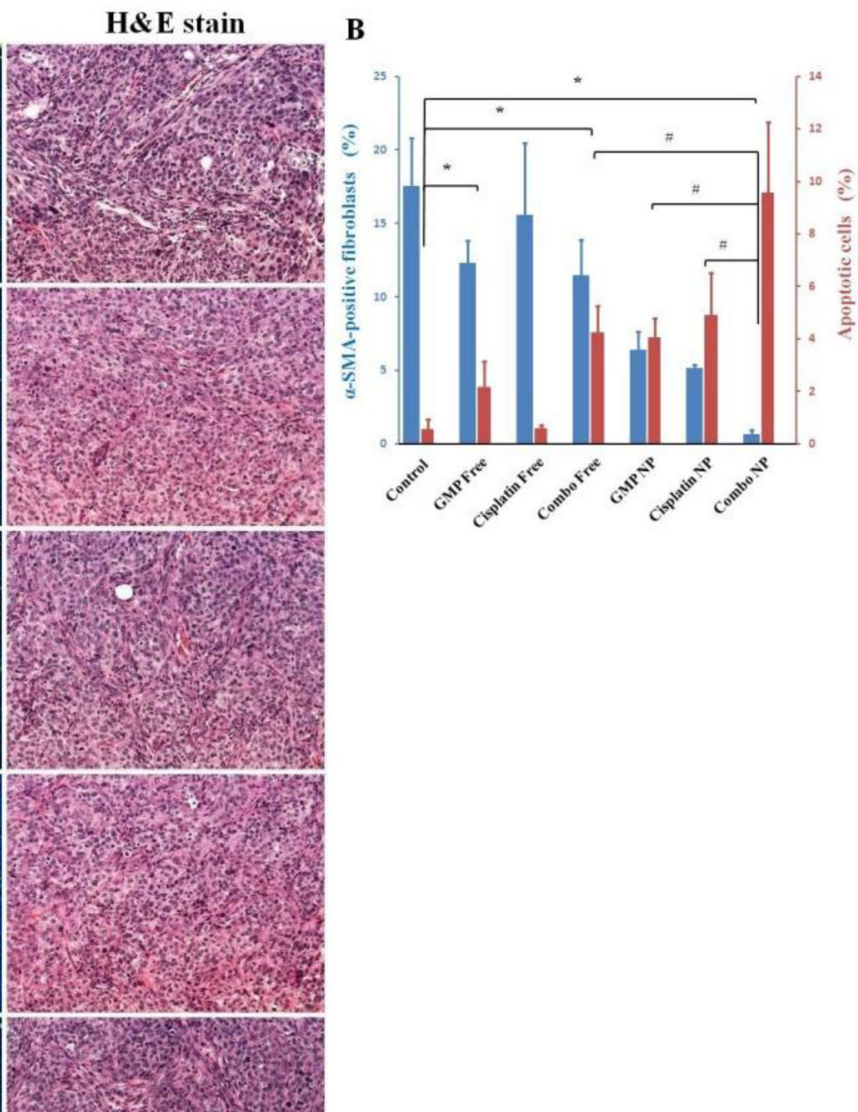

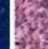
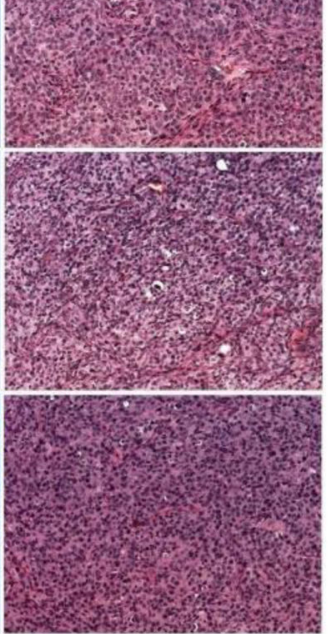

Fig. 4.

Effects of different treatments on the induction of apoptosis in tumor, the inhibition of fibroblast growth and tumor tissue H\&E stain (20x) (A) and quantitative results expressed as the percentage of total cell number (B). * $\mathrm{p}<0.05$ vs. Control; \# $\mathrm{p}<0.05$ vs.

Combo NP. 
A
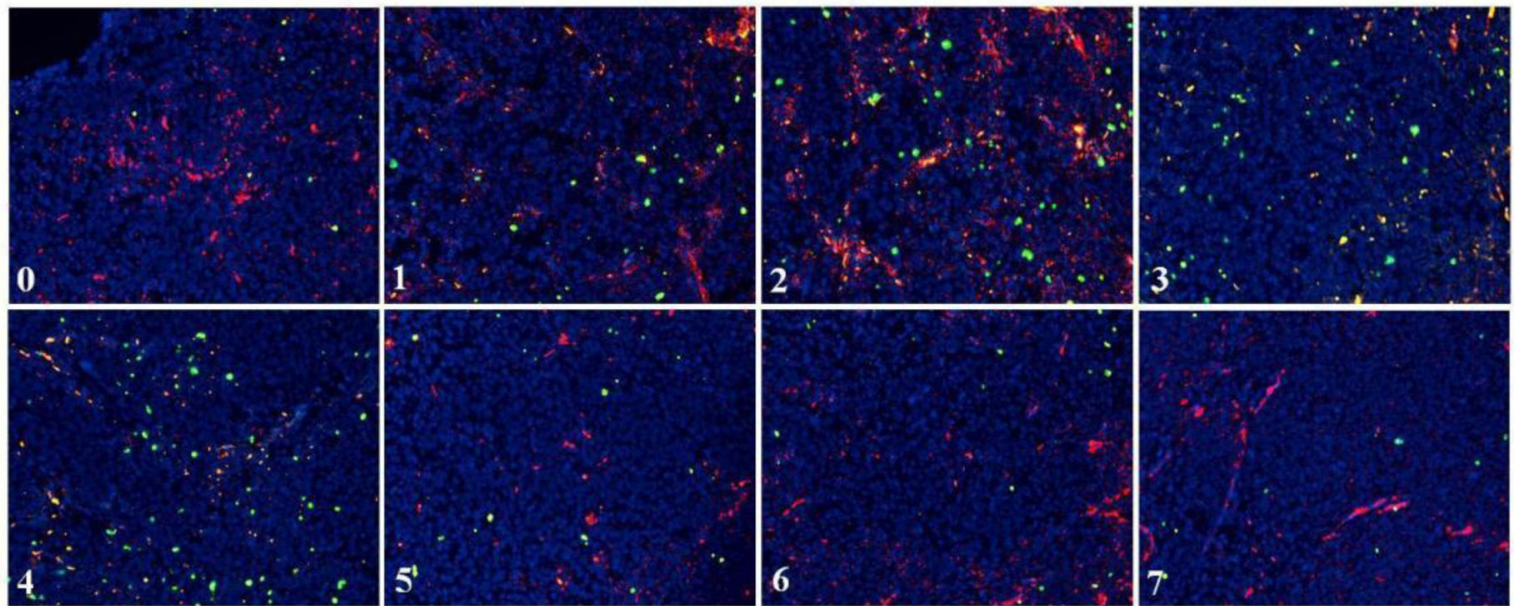

B

C

D

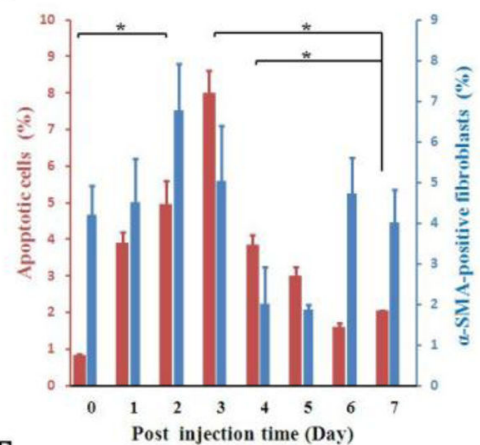

$\mathbf{E}$
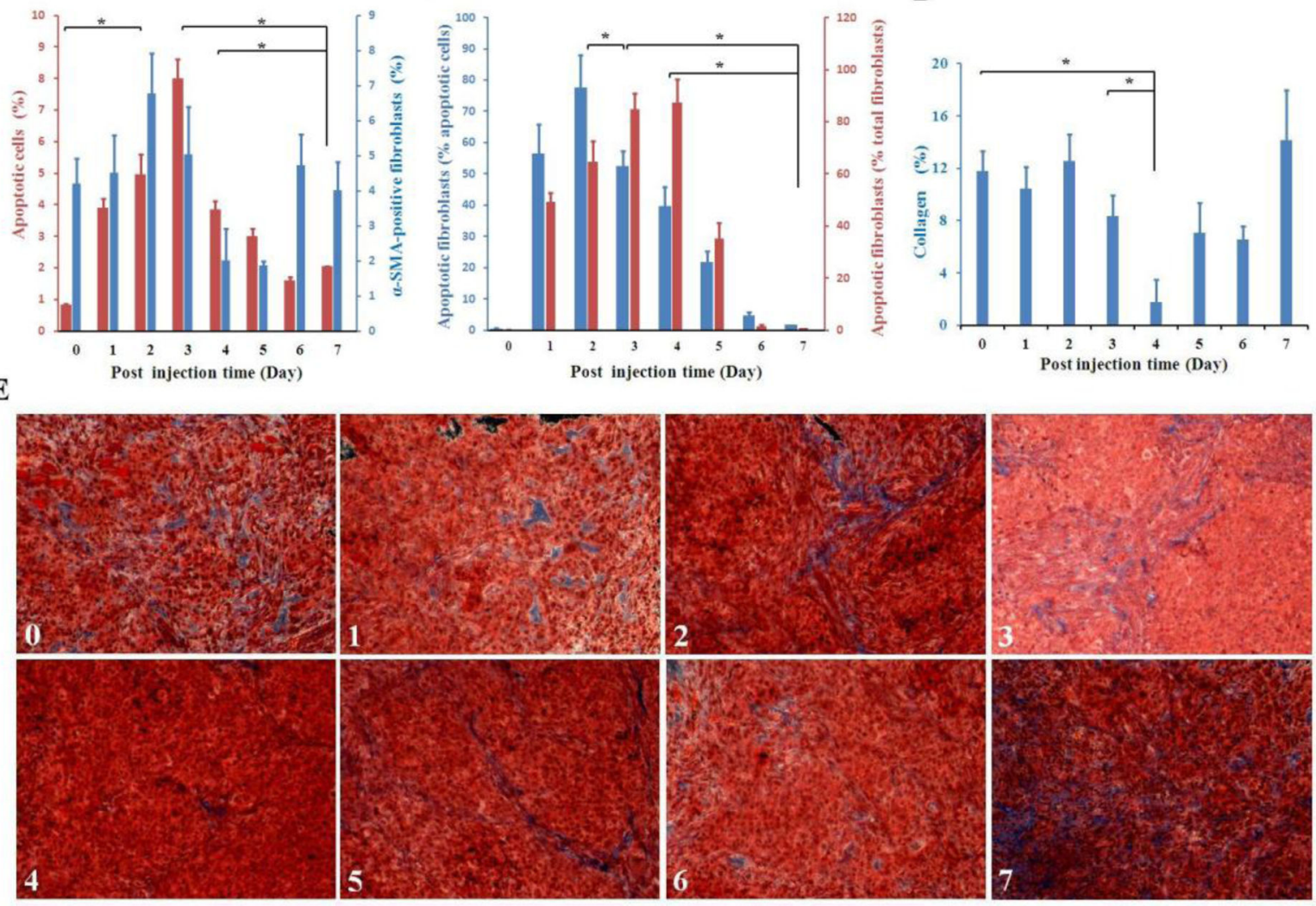

Fig. 5.

Immunofluorescence double staining for SMA-positive fibroblasts (red), TUNEL (green) and apoptotic fibroblasts (yellow) (A); quantitative results for TUNEL-positive cells and a-SMA-positive fibroblasts (B); quantitative results for apoptotic fibroblasts expressed as the percentage of total apoptotic cells and fibroblasts (C); quantitative results for collagen expressed by the area (\%) (D); Masson's trichrome stain for collagen (blue) (E) in tumor-bearing mice treated with a single injection of the Combo NP. Tumors were excised on Day 0 and every $24 \mathrm{~h}$ for 7 days for analysis and expressed as 0 (Day 0) to 7 (Day 7 ) in the image.

$$
* \mathrm{p}<0.05 \text {. }
$$




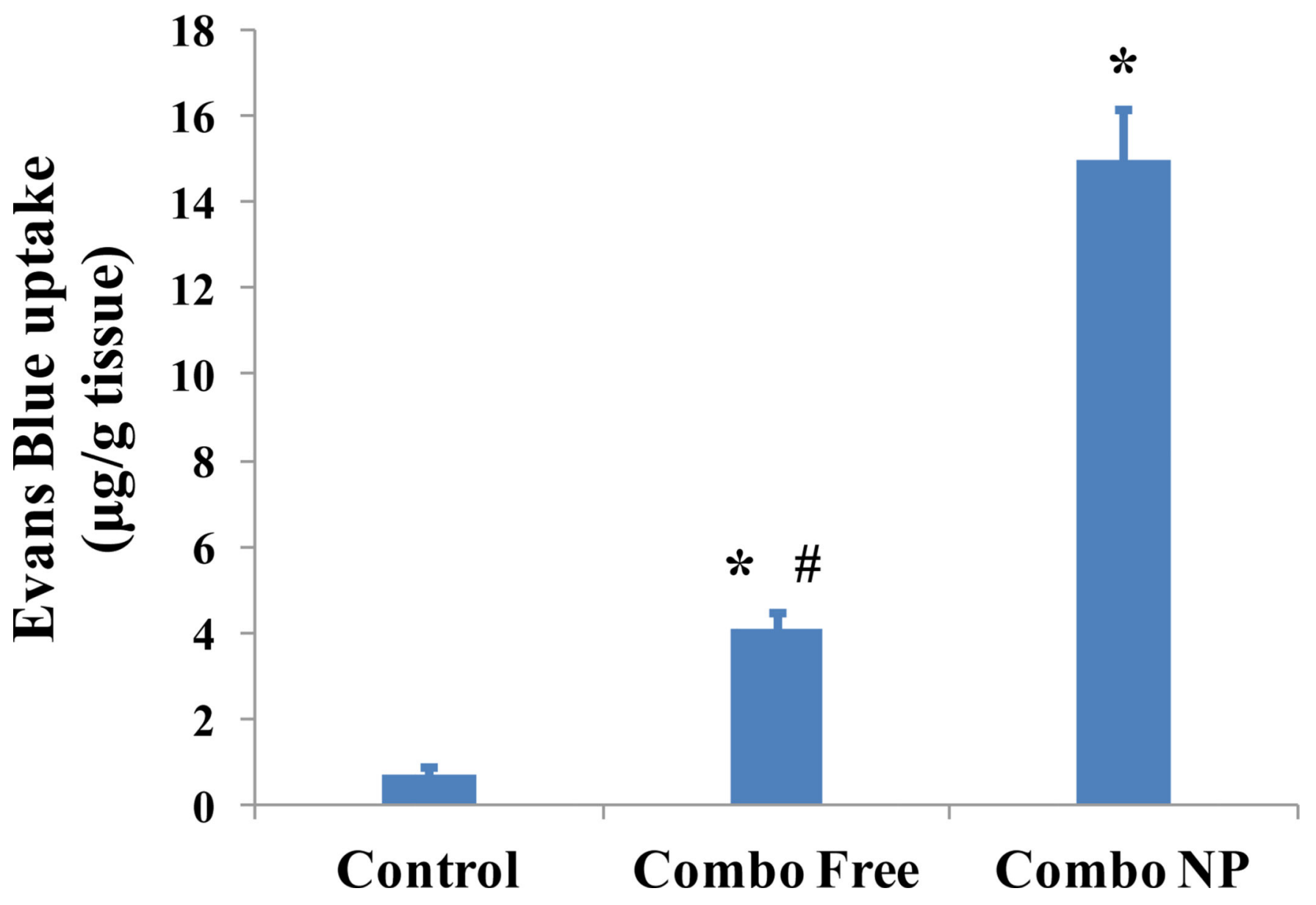

Fig. 6.

Evans Blue staining of mice to determine tumor vessel leakiness. Single intravenous injection was performed for each treatment with the GMP dose at $16 \mathrm{mg} / \mathrm{kg}$ and Cisplatin at $1.6 \mathrm{mg} / \mathrm{kg}$. Twenty-four hours later, mice were injected with $100 \mathrm{mg} / \mathrm{kg}$ of Evans blue. After $30 \mathrm{~min}$, the mice were sacrificed, and samples from the tumors were removed. The concentration of Evans Blue was then determined spectrophotometrically. Results are shown as the mean weight of Evans Blue per gram tissue. * p $<0.05$ vs. Control; \# $\mathrm{p}<0.05$ vs. Combo NP. $\mathrm{n}=3$. 
Table 1

Effect of GMP NP, Cisplatin NP, Combo Free and Combo NP on serum ALT, AST, BUN and creatinine levels

\begin{tabular}{lllll}
\hline Treatment & ALT (U/L) & AST (U/L) & $\begin{array}{l}\text { BUN } \\
(\mathbf{m m o l} / \mathbf{L})\end{array}$ & $\begin{array}{l}\text { Creatinine } \\
(\mathbf{m g} / \mathbf{d L})\end{array}$ \\
\hline Control & $60.7 \pm 15.3$ & $191 \pm 13.7$ & $5.2 \pm 0.4$ & 0.2 \\
GMP NP & $59.6 \pm 8.7$ & $98 \pm 11.6$ & $6.4 \pm 1.6$ & $0.2 \pm 0.1$ \\
Cisplatin NP & $66.3 \pm 1.5$ & $171 \pm 8.2$ & $7.2 \pm 1.2$ & 0.2 \\
Combo Free & $44.3 \pm 10.2$ & $230 \pm 8.9$ & 5.7 & 0.2 \\
Combo NP & $58.3 \pm 6.8$ & $252 \pm 10.3$ & $5.3 \pm 0.4$ & $0.2 \pm 0.1$ \\
\hline
\end{tabular}

